\title{
Methods for Solving Generalized Nash Equilibrium
}

\author{
Biao Qu and Jing Zhao \\ School of Management, Qufu Normal University, Rizhao, Shandong 276826, China \\ Correspondence should be addressed to Biao Qu; qubiao001@163.com
}

Received 12 October 2012; Revised 5 January 2013; Accepted 17 January 2013

Academic Editor: Ya Ping Fang

Copyright (C) 2013 B. Qu and J. Zhao. This is an open access article distributed under the Creative Commons Attribution License, which permits unrestricted use, distribution, and reproduction in any medium, provided the original work is properly cited.

\begin{abstract}
The generalized Nash equilibrium problem (GNEP) is an extension of the standard Nash equilibrium problem (NEP), in which each player's strategy set may depend on the rival player's strategies. In this paper, we present two descent type methods. The algorithms are based on a reformulation of the generalized Nash equilibrium using Nikaido-Isoda function as unconstrained optimization. We prove that our algorithms are globally convergent and the convergence analysis is not based on conditions guaranteeing that every stationary point of the optimization problem is a solution of the GNEP.
\end{abstract}

\section{Introduction}

The generalized Nash equilibrium problem (GNEP for short) is an extension of the standard Nash equilibrium problem (NEP for short), in which the strategy set of each player depends on the strategies of all the other players as well as on his own strategy. The GNEP has recently attracted much attention due to its applications in various fields like mathematics, computer science, economics, and engineering [1-11]. For more details, we refer the reader to a recent survey paper by Facchinei and Kanzow [3] and the references therein.

Let us first recall the definition of the GNEP. There are $N$ players labelled by an integer $v=1, \ldots, N$. Each player $v$ controls the variables $x^{v} \in R^{n_{v}}$. Let $x=\left(x^{1} \cdots x^{n}\right)^{T}$ be the vector formed by all these decision variables, where $n:=$ $n_{1}+n_{2}+\cdots+n_{v}$. To emphasize the $v$ th player variable within the vector $x$, we sometimes write $x=\left(x^{v}, x^{-v}\right)^{T} \in R^{n}$, where $x^{-v}$ denotes all the other player's variables. In the games, each player controls the variables $x^{v}$ and tries to minimize a cost function $\theta_{v}\left(x^{v}, x^{-v}\right)$ subjects to the constraint $\left(x^{v}, x^{-v}\right)^{T} \in X$ with $x^{-v}$ given as exogenous, where $X$ is a common strategy set. A vector $x^{*}:=\left(x^{*, 1}, \ldots, x^{*, N}\right)^{T}$ is called a solution of the GNEP or a generalized Nash equilibrium, if for each player $v=1, \ldots, N, x^{*, v}$ solves the following optimization problem with $x^{*,-v}$ being fixed:

$$
\begin{aligned}
& \min _{x^{v}} \theta_{v}\left(x^{v}, x^{*,-v}\right), \\
& \text { s.t. }\left(x^{v}, x^{*,-v}\right) \in X .
\end{aligned}
$$

If $X$ is defined as the Cartesian product of certain sets $X_{v} \in R^{n_{v}}$, that is, $X=X_{1} \times X_{2} \times \cdots \times X_{N}$, then the GNEP reduces to the standard Nash equilibrium problem.

Throughout this paper, we can make the following assumption.

Assumption 1. (a) The set $X$ is nonempty, closed, and convex. (b) The utility function $\theta_{v}$ is continuously differentiable and, as a function of $x^{v}$ alone, convex.

A basic tool for both the theoretical and the numerical solution of (generalized) Nash equilibrium problems is the Nikaido-Isoda function defined as

$$
\Psi(x, y)=\sum_{v=1}^{N}\left[\theta_{v}\left(x^{v}, x^{-v}\right)-\theta_{v}\left(y^{v}, x^{-v}\right)\right] .
$$

Sometimes also the name Ky-Fan function can be found in the literature, see $[12,13]$. In the following, we state a definition which we have taken from [9].

Definition 1. $x^{*}$ is a normalized Nash equilibrium of the GNEP, if $\max _{y} \Psi\left(x^{*}, y\right)=0$ holds, where $\Psi(x, y)$ denotes the Nikaido-Isoda function defined as (2).

In order to overcome the nondifferentiable property of the mapping $\Psi(x, y)$, von Heusinger and Kanzow [8] used a simple regularization of the Nikaido-Isoda function. For 
a parameter $\alpha>0$, the following regularized Nikaido-Isoda function was considered:

$$
\Psi_{\alpha}(x, y)=\sum_{v=1}^{N}\left[\theta_{v}\left(x^{v}, x^{-v}\right)-\theta_{v}\left(y^{v}, x^{-v}\right)\right]-\frac{\alpha}{2}\|x-y\|^{2} .
$$

Since under the given Assumption $1, \Psi_{\alpha}(x, y)$ is strongly concave in $y$, the maximization problem

$$
\begin{gathered}
\max _{y} \Psi_{\alpha}(x, y), \\
\text { s.t. } y \in X
\end{gathered}
$$

has a unique solution for each $x$, denoted by $y_{\alpha}(x)$.

The corresponding value function is then defined by

$$
V_{\alpha}(x)=\max _{y} \Psi_{\alpha}(x, y)=\Psi_{\alpha}\left(x, y_{\alpha}(x)\right)
$$

Let $\beta>\alpha>0$ be a given parameter. The corresponding value function is then defined by

$$
V_{\beta}(x)=\max _{y} \Psi_{\beta}(x, y)=\Psi_{\beta}\left(x, y_{\beta}(x)\right) .
$$

Define

$$
V_{\alpha \beta}(x)=V_{\alpha}(x)-V_{\beta}(x) .
$$

In [8], the following important properties of the function $V_{\alpha \beta}(x)$ have been proved.

Theorem 2. The following statements hold:

(a) $V_{\alpha \beta}(x) \geq 0$ for any $x \in R^{n}$;

(b) $x^{*}$ is a normalized Nash equilibrium of the GNEP if and only if $V_{\alpha \beta}\left(x^{*}\right)=0$;

(c) $V_{\alpha \beta}(x)$ is continuously differentiable on $R^{n}$ and that

$$
\begin{aligned}
\nabla & V_{\alpha \beta}(x) \\
= & \nabla V_{\alpha}(x)-\nabla V_{\beta}(x) \\
= & \sum_{v=1}^{N}\left[\nabla \theta_{v}\left(y_{\beta}(x)^{v}, x^{-v}\right)-\nabla \theta_{v}\left(y_{\alpha}(x)^{v}, x^{-v}\right)\right] \\
& +\left(\begin{array}{c}
\nabla_{x^{1}} \theta_{1}\left(y_{\alpha}(x)^{1}, x^{-1}\right)-\nabla_{x^{1}} \theta_{1}\left(y_{\beta}(x)^{1}, x^{-1}\right) \\
\vdots \\
\nabla_{x^{N}} \theta_{N}\left(y_{\alpha}(x)^{N}, x^{-N}\right)-\nabla_{x^{N}} \theta_{N}\left(y_{\beta}(x)^{N}, x^{-N}\right)
\end{array}\right) \\
& -\alpha\left(x-y_{\alpha}(x)\right)+\beta\left(x-y_{\beta}(x)\right) .
\end{aligned}
$$

From Theorem 2, we know that the normalized Nash equilibrium of the GNEP is precisely the global minima of the smooth unconstrained optimization problem (see [5]) as

$$
\min _{x \in R^{n}} V_{\alpha \beta}(x)
$$

with zero optimal value.
In this paper, we develop two new descent methods for finding a normalized Nash equilibrium of the GNEP by solving the optimization problem (9). The key to our methods is a strategy for adjusting $\alpha$ and $\beta$ when a stationary point of $\mathrm{V}_{\alpha \beta}(x)$ is not a solution of the GNEP. We will show that our algorithms are globally convergent to a normalized Nash equilibrium under appropriate assumption on the cost function, which is not stronger than the one considered in [8].

The organization of the paper is as follows. In Section 2, we state the main assumption underlying our algorithms and present some examples of the GNEP satisfying it. In Section 3, we derive some useful properties of the function $V_{\alpha \beta}(x)$. In Section 4, we formally state our algorithms and prove that they are both globally convergent to a normalized Nash equilibrium.

\section{Main Assumption}

In order to construct algorithms and guarantee the convergence of them, we give the following assumption.

Assumption 2. For any $\beta>\alpha>0$ and $x \in R^{n}$, if $y_{\alpha}(x) \neq y_{\beta}(x)$, we have

$$
\begin{aligned}
\sum_{v=1}^{N}\left(\nabla \theta_{v}\left(y_{\beta}(x)^{v}, x^{-v}\right)-\nabla \theta_{v}\left(y_{\alpha}(x)^{v}, x^{-v}\right)\right)^{T} \\
\cdot\left(y_{\beta}(x)-y_{\alpha}(x)\right) \\
\geq \sum_{v=1}^{N}\left(\nabla_{x^{v}} \theta_{v}\left(y_{\beta}(x)^{v}, x^{-v}\right)-\nabla_{x^{v}} \theta_{v}\left(y_{\alpha}(x)^{v}, x^{-v}\right)\right)^{T} \\
\cdot\left(y_{\beta}(x)^{v}-y_{\alpha}(x)^{v}\right) .
\end{aligned}
$$

We next consider three examples which satisfy Assumption 2.

Example 3. Let us consider the case in which all the cost functions are separable, that is,

$$
\theta_{v}(x)=f_{v}\left(x^{v}\right)+g_{v}\left(x^{-v}\right)
$$

where $f_{v}: R^{n_{v}} \rightarrow R$ is convex and $g_{v}: R^{n-n_{v}} \rightarrow R$. A simple calculation shows that, for any $y \in R^{n}$, we have

$$
\begin{aligned}
\sum_{v=1}^{N}\left(\nabla_{x^{v}} \theta_{v}\left(y_{\beta}(x)^{v}, x^{-v}\right)-\nabla_{x^{v}} \theta_{v}\left(y_{\alpha}(x)^{v}, x^{-v}\right)\right)^{T} \\
\cdot\left(y_{\beta}(x)^{v}-y_{\alpha}(x)^{v}\right) \\
=\sum_{v=1}^{N}\left(\nabla f_{v}\left(y_{\beta}(x)^{v}\right)-\nabla f_{v}\left(y_{\alpha}(x)^{v}\right)\right)^{T}\left(y_{\beta}(x)^{v}-y_{\alpha}(x)^{v}\right), \\
\sum_{v=1}^{N}\left(\nabla \theta_{v}\left(y_{\beta}(x)^{v}, x^{-v}\right)-\nabla \theta_{v}\left(y_{\alpha}(x)^{v}, x^{-v}\right)\right)^{T} \\
\cdot\left(y_{\beta}(x)^{v}-y_{\alpha}(x)^{v}\right) \\
=\sum_{v=1}^{N}\left(\nabla f_{v}\left(y_{\beta}(x)^{v}\right)-\nabla f_{v}\left(y_{\alpha}(x)^{v}\right)\right)^{T}\left(y_{\beta}(x)^{v}-y_{\alpha}(x)^{v}\right) .
\end{aligned}
$$

Hence Assumption 2 holds. 
Example 4. Consider the case where the cost function $\theta_{v}(x)$ is quadratic, that is,

$$
\theta_{v}(x)=\frac{1}{2}\left(x^{v}\right)^{T} A_{v v} x^{v}+\sum_{\mu=1, \mu \neq v}^{N}\left(x^{v}\right)^{T} A_{v \mu} x^{\mu}
$$

for $v=1, \ldots, N$. We have

$$
\begin{aligned}
& \sum_{v=1}^{N}\left(\nabla_{x^{v}} \theta_{v}\left(y_{\beta}(x)^{v}, x^{-v}\right)-\nabla_{x^{v}} \theta_{v}\left(y_{\alpha}(x)^{v}, x^{-v}\right)\right)^{T} \\
& \cdot\left(y_{\beta}(x)^{v}-y_{\alpha}(x)^{v}\right) \\
& =\sum_{v=1}^{N}\left\langle y_{\beta}(x)^{v}-y_{\alpha}(x)^{v}, A_{v v}\left(y_{\beta}(x)^{v}-y_{\alpha}(x)^{v}\right)\right\rangle \text {, } \\
& \sum_{v=1}^{N}\left(\nabla \theta_{v}\left(y_{\beta}(x)^{v}, x^{-v}\right)-\nabla \theta_{v}\left(y_{\alpha}(x)^{v}, x^{-v}\right)\right)^{T} \\
& \cdot\left(y_{\beta}(x)-y_{\alpha}(x)\right) \\
& =\left\langle y_{\beta}(x)-y_{\alpha}(x),\right. \\
& \left.\left(\begin{array}{cccc}
A_{11} & A_{21} & \cdots & A_{1 N} \\
A_{21} & A_{22} & \cdots & A_{2 N} \\
\vdots & \vdots & \ddots & \vdots \\
A_{1 N} & A_{2 N} & \cdots & A_{N N}
\end{array}\right) y_{\beta}(x)-y_{\alpha}(x)\right\rangle
\end{aligned}
$$

Therefore, if the matrix

$$
\left(\begin{array}{cccc}
0 & A_{12} & \cdots & A_{1 N} \\
A_{21} & 0 & \cdots & A_{2 N} \\
\vdots & \vdots & \ddots & \vdots \\
A_{N 1} & A_{N 2} & \cdots & 0
\end{array}\right)
$$

is positive semidefinite, Assumption 2 is satisfied.

In the following example, we show the relationship between our assumption and the one considered in [8] as follows.

For any $\beta>\alpha>0$, a given $x \in R^{n}$ with $y_{\alpha}(x) \neq y_{\beta}(x)$, the inequality

$$
\begin{aligned}
\sum_{v=1}^{N}( & \left.\nabla \theta_{v}\left(y_{\beta}(x)^{v}, x^{-v}\right)-\nabla \theta_{v}\left(y_{\alpha}(x)^{v}, x^{-v}\right)\right)^{T} \\
\quad & \times\left(y_{\beta}(x)-y_{\alpha}(x)\right)>0
\end{aligned}
$$

holds.
Example 5. Consider the GNEP with $N=2$ as

$$
X=\left\{x \in R^{n}: x^{1} \geq 1, x^{2} \geq 1, x^{1}+x^{2} \leq 10\right\}
$$

and the cost function $\theta_{1}(x)=x^{1} x^{2}$ and $\theta_{2}(x)=-x^{1} x^{2}$. The point $x^{*}=(1,9)^{T}$ is the unique normalized Nash equilibrium. For any $y \in R^{2}$, we have

$$
\begin{aligned}
& \sum_{v=1}^{N}\left(\nabla \theta_{v}\left(y_{\beta}(x)^{v}, x^{-v}\right)-\nabla \theta_{v}\left(y_{\alpha}(x)^{v}, x^{-v}\right)\right)^{T} \\
& \cdot\left(y_{\beta}(x)-y_{\alpha}(x)\right)=0, \\
& \sum_{v=1}^{N}\left(\nabla_{x^{v}} \theta_{v}\left(y_{\beta}(x)^{v}, x^{-v}\right)-\nabla_{x^{v}} \theta_{v}\left(y_{\alpha}(x)^{v}, x^{-v}\right)\right)^{T} \\
& \cdot\left(y_{\beta}(x)^{v}-y_{\alpha}(x)^{v}\right)=0 .
\end{aligned}
$$

Therefore Assumption 2 holds, but (16) does not hold for any $\beta>\alpha>0$.

\section{Properties of $V_{\alpha \beta}(x)$}

Lemma 6. For any $\beta>\alpha>0$ and $x \in R^{n}$, we have

$$
\begin{aligned}
& V_{\alpha \beta}(x) \geq \frac{\beta-\alpha}{2}\left\|x-y_{\beta}(x)\right\|^{2}+\frac{\alpha}{2}\left\|y_{\alpha}(x)-y_{\beta}(x)\right\|^{2}, \\
& V_{\alpha \beta}(x) \leq \frac{\beta-\alpha}{2}\left\|x-y_{\alpha}(x)\right\|^{2}-\frac{\beta}{2}\left\|y_{\alpha}(x)-y_{\beta}(x)\right\|^{2} .
\end{aligned}
$$

Proof. Since $y_{\alpha}(x)$ satisfies the optimality condition, then

$$
\begin{gathered}
\sum_{v=1}^{N}\left[\nabla_{x^{v}} \theta_{v}\left(y_{\alpha}(x)^{v}, x^{-v}\right)-\alpha\left(x^{v}-y_{\alpha}(x)^{v}\right)\right]^{T} \\
\cdot\left(y_{\beta}(x)^{v}-y_{\alpha}(x)^{v}\right) \geq 0 .
\end{gathered}
$$

In a similar way, it follows that $y_{\beta}(x)$ satisfies

$$
\begin{gathered}
\sum_{v=1}^{N}\left[\nabla_{x^{v}} \theta_{v}\left(y_{\beta}(x)^{v}, x^{-v}\right)-\beta\left(x^{v}-y_{\beta}(x)^{v}\right)\right]^{T} \\
\cdot\left(y_{\alpha}(x)^{v}-y_{\beta}(x)^{v}\right) \geq 0 .
\end{gathered}
$$

Since $\theta_{v}(x)$ as a function of $x^{v}$ alone is convex, we have

$$
\begin{gathered}
\sum_{v=1}^{N}\left[\theta_{v}\left(y_{\beta}(x)^{v}, x^{-v}\right)-\theta_{v}\left(y_{\alpha}(x)^{v}, x^{-v}\right)\right] \\
-\alpha\left(x-y_{\alpha}(x)\right)^{T}\left(y_{\beta}(x)-y_{\alpha}(x)\right) \geq 0, \\
\sum_{v=1}^{N}\left[\theta_{v}\left(y_{\alpha}(x)^{v}, x^{-v}\right)-\theta_{v}\left(y_{\beta}(x)^{v}, x^{-v}\right)\right] \\
-\beta\left(x-y_{\beta}(x)\right)^{T}\left(y_{\alpha}(x)-y_{\beta}(x)\right) \geq 0
\end{gathered}
$$


respectively. Thus, using the definition of $V_{\alpha \beta}(x)$ and (23), we have

$$
\begin{aligned}
V_{\alpha \beta}(x)= & \sum_{v=1}^{N}\left[\theta_{v}\left(y_{\alpha}(x)^{v}, x^{-v}\right)-\theta_{v}\left(y_{\beta}(x)^{v}, x^{-v}\right)\right] \\
& +\frac{\beta}{2}\left\|x-y_{\beta}(x)\right\|^{2}-\frac{\alpha}{2}\left\|x-y_{\alpha}(x)\right\|^{2} \\
\geq & \alpha\left(x-y_{\alpha}(x)\right)^{T}\left(y_{\beta}(x)-y_{\alpha}(x)\right) \\
& +\frac{\beta}{2}\left\|x-y_{\beta}(x)\right\|^{2}-\frac{\alpha}{2}\left\|x-y_{\alpha}(x)\right\|^{2} \\
= & \alpha\left(x-y_{\alpha}(x)\right)^{T}\left(y_{\beta}(x)-y_{\alpha}(x)\right)+\frac{\beta}{2}\left\|x-y_{\beta}(x)\right\|^{2} \\
& -\frac{\alpha}{2}\left\|x-y_{\beta}(x)+y_{\beta}(x)-y_{\alpha}(x)\right\|^{2} \\
= & \frac{\beta-\alpha}{2}\left\|x-y_{\beta}(x)\right\|^{2}+\frac{\alpha}{2}\left\|y_{\alpha}(x)-y_{\beta}(x)\right\|^{2} .
\end{aligned}
$$

Similarly, using the definition of $V_{\alpha \beta}(x)$ and (24), we have

$$
V_{\alpha \beta}(x) \leq \frac{\beta-\alpha}{2}\left\|x-y_{\alpha}(x)\right\|^{2}-\frac{\beta}{2}\left\|y_{\alpha}(x)-y_{\beta}(x)\right\|^{2} \text {. }
$$

The proof is complete.

Lemma 7. Assume $X$ is bounded. For any $\beta>\alpha>0$ and $x \in R^{n}$, we have

$$
\limsup _{\beta^{\prime} \rightarrow \infty, \alpha^{\prime} \rightarrow 0} \frac{V_{\alpha^{\prime} \beta^{\prime}}(x)}{\beta^{\prime}-\alpha^{\prime}} \leq \frac{V_{\alpha \beta}(x)}{\beta-\alpha} .
$$

Proof. We have from (19) that

$$
\begin{aligned}
& \frac{2 V_{\alpha \beta}(x)}{\beta-\alpha} \\
& \quad \geq\left\|x-y_{\beta}(x)\right\|^{2}+\frac{\alpha}{\beta-\alpha}\left\|y_{\alpha}(x)-y_{\beta}(x)\right\|^{2} \\
& \quad \geq\left\|x-y_{\beta}(x)\right\|^{2} .
\end{aligned}
$$

By the definition of $V_{\alpha \beta}(x)$, we have

$$
\begin{aligned}
& \frac{2 V_{\alpha^{\prime} \beta^{\prime}}}{\beta^{\prime}-\alpha^{\prime}} \\
& =\frac{2 \sum_{v=1}^{N}\left[\theta_{v}\left(y_{\beta^{\prime}}(x)^{v}, x^{-v}\right)-\theta_{v}\left(y_{\alpha^{\prime}}(x)^{v}, x^{-v}\right)\right]}{\beta^{\prime}-\alpha^{\prime}} \\
& -\frac{\alpha^{\prime}\left\|x-y_{\alpha^{\prime}}(x)\right\|^{2}+\beta^{\prime}\left\|x-y_{\beta^{\prime}}(x)\right\|^{2}}{\beta^{\prime}-\alpha^{\prime}} .
\end{aligned}
$$
that

Since $y_{\alpha}(x) \in X, y_{\beta}(x) \in X$ and $X$ is bounded, we get

$$
\limsup _{\beta^{\prime} \rightarrow \infty, \alpha^{\prime} \rightarrow 0} \frac{V_{\alpha^{\prime} \beta^{\prime}}(x)}{\beta^{\prime}-\alpha^{\prime}} \leq \frac{1}{2}\left\|x-y_{\beta}(x)\right\|^{2} \leq \frac{V_{\alpha \beta}}{\beta-\alpha} .
$$

This completes the proof.
Equation (8) and Assumption 2 yield

$$
\begin{aligned}
& V_{\alpha \beta}(x)^{T}\left(y_{\beta}(x)-y_{\alpha}(x)\right) \\
& =\sum_{v=1}^{N}\left[\nabla \theta_{v}\left(y_{\beta}(x)^{v}, x^{-v}\right)-\nabla \theta_{v}\left(y_{\alpha}(x)^{v}, x^{-v}\right)\right]^{T} \\
& \cdot\left(y_{\beta}(x)-y_{\alpha}(x)\right) \\
& +\left(\begin{array}{c}
\nabla_{x^{1}} \theta_{1}\left(y_{\alpha}(x)^{1}, x^{-1}\right)-\nabla_{x^{1}} \theta_{1}\left(y_{\beta}(x)^{1}, x^{-1}\right) \\
\vdots \\
\nabla_{x^{N}} \theta_{N}\left(y_{\alpha}(x)^{N}, x^{-N}\right)-\nabla_{x^{N}} \theta_{N}\left(y_{\beta}(x)^{N}, x^{-N}\right)
\end{array}\right)^{T} \\
& \cdot\left(y_{\beta}(x)-y_{\alpha}(x)\right) \\
& -\left[\alpha\left(x-y_{\alpha}(x)\right)-\beta\left(x-y_{\beta}(x)\right)\right]^{T} \\
& \cdot\left(y_{\beta}(x)-y_{\alpha}(x)\right) \\
& \geq-\left[\alpha\left(x-y_{\alpha}(x)\right)-\beta\left(x-y_{\beta}(x)\right)\right]^{T} \\
& \cdot\left(y_{\beta}(x)-y_{\alpha}(x)\right) \\
& =: e_{\alpha \beta}(x) \geq 0 \text {, }
\end{aligned}
$$

where nonnegativity of $e_{\alpha \beta}(x)$ follows from the inequalities (23) and (24). In particular, either $e_{\alpha \beta}(x)$ is above a tolerance $\varepsilon>0$, in which case $y_{\alpha}(x)-y_{\beta}(x)$ is a direction of sufficient descent for $V_{\alpha \beta}(x)$ at $x$ or else, as we show in the lemma below, and $x$ is an approximate solution of the GNEP with accuracy depending on $\varepsilon, \alpha, \beta$. This result will lead to our methods.

Lemma 8. For any $\beta>\alpha>0$ and $x \in R^{n}$, we have

$$
\begin{gathered}
\left\|x-y_{\beta}(x)\right\| \leq \sqrt{\frac{2 V_{\alpha \beta}(x)}{\beta-\alpha}}, \\
\gamma_{\alpha \beta}(x) \leq V_{\alpha}(x) \leq \gamma_{\alpha \beta}(x)+e_{\alpha \beta}(x)+\frac{\alpha}{2}\left\|y_{\alpha}(x)-y_{\beta}(x)\right\|^{2},
\end{gathered}
$$

where $\gamma_{\alpha \beta}(x)=\sum_{v=1}^{N}\left[\theta_{v}\left(x^{v}, x^{-v}\right)-\theta_{v}\left(y_{\beta}(x)^{v}, x^{-v}\right)\right]-$ $(\alpha / 2)\left\|x-y_{\beta}(x)\right\|^{2}$.

Proof. Inequality (32) follows immediately from (19) in Lemma 6.

The definition of $V_{\alpha}(x)$ implies that

$$
\begin{aligned}
V_{\alpha}(x) \geq \sum_{v=1}^{N}[ & \left.\theta_{v}\left(x^{v}, x^{-v}\right)-\theta_{v}\left(y_{\beta}(x)^{v}, x^{-v}\right)\right] \\
- & \frac{\alpha}{2}\left\|x-y_{\beta}(x)\right\|^{2},
\end{aligned}
$$

which proves the first inequality in (33).

Since $e_{\alpha \beta}(x)$ is the sum of the nonnegative quantity $\left(\sum_{v=1}^{N}\left[\theta_{v}\left(y_{\beta}(x)^{v}, x^{-v}\right)-\theta_{v}\left(y_{\alpha}(x)^{v}, x^{-v}\right)\right]-\alpha\left(x-y_{\alpha}(x)\right)^{T}\right.$ $\left.\cdot\left(y_{\beta}(x)-y_{\alpha}(x)\right)\right)$ with another nonnegative quantity (see (23) and (24)), we have

$$
\begin{array}{r}
e_{\alpha \beta}(x) \geq \sum_{v=1}^{N}\left[\theta_{v}\left(y_{\beta}(x)^{v}, x^{-v}\right)-\theta_{v}\left(y_{\alpha}(x)^{v}, x^{-v}\right)\right] \\
-\alpha\left(x-y_{\alpha}(x)\right)^{T}\left(y_{\beta}(x)-y_{\alpha}(x)\right) .
\end{array}
$$


Thus,

$$
V_{\alpha}(x) \leq \gamma_{\alpha \beta}(x)+e_{\alpha \beta}(x)+\frac{\alpha}{2}\left\|y_{\alpha}(x)-y_{\beta}(x)\right\|^{2},
$$

which is the second inequality in (33). This completes the proof.

\section{Two Methods for Solving the GNEP}

In this section, we introduce two methods for solving the GNEP, motivated by the D-gap function scheme for solving monotone variational inequalities $[14,15]$. We first formally describe our methods below and then analyze their convergence using Lemma 8.

Algorithm 9. Choose an arbitrary initial point $x^{0} \in R^{n}$, and any $\beta_{0}>\alpha_{0}>0$. Choose any sequences of numbers $\varepsilon^{k}>$ $0, \eta_{k} \geq 0, \lambda_{k} \in[0,1), k=1,2, \ldots$, such that

$$
\lim _{k \rightarrow \infty} \varepsilon^{k}=\lim _{k \rightarrow \infty} \frac{\eta_{k}}{1-\lambda_{k}}=0, \quad \sum_{k=1}^{\infty}\left(1-\lambda_{k}\right)=\infty .
$$

For $k=1,2, \ldots$, we iterate the following. Iteration $k$. Choose any $0<\alpha_{k} \leq(1 / 2) \alpha_{k-1}$. Choose any $\beta_{k} \geq$ $2 \beta_{k-1}$ and $\bar{x}^{k} \in R^{n}$ satisfying

$$
\frac{V_{\alpha_{k} \beta_{k}}\left(\bar{x}^{k}\right)}{\beta_{k}-\alpha_{k}} \leq \eta_{k}+\lambda_{k} \frac{V_{\alpha_{k-1} \beta_{k-1}}\left(x^{k-1}\right)}{\beta_{k-1}-\alpha_{k-1}} .
$$

Apply a descent method to the unconstrained minimization of the function $V_{\alpha_{k} \beta_{k}}$, with $\bar{x}^{k}$ as the starting point and using $y_{\alpha_{k}}-y_{\beta_{k}}$ as a safeguard descent direction at $x$, until the method generates an $x \in R^{n}$ satisfying $e_{\alpha_{k} \beta_{k}}(x) \leq \varepsilon^{k}$. The resulting $x$ is denoted by $x^{k}$.

Theorem 10. Assume $X$ is bounded. Let $\left\{x^{k}, \alpha_{k}, \beta_{k}, \varepsilon_{k}\right.$, $\left.\eta_{k}, \lambda_{k}\right\}_{k=0,1,2, \ldots}$ be generated by Algorithm 9. Then $\left\{x^{k}\right\}$ is bounded; $\beta_{k} \rightarrow \infty ; \alpha_{k} \rightarrow 0$; and every cluster point of $\left\{x^{k}\right\}$ is a normalized Nash equilibrium of the GNEP.

Proof. Denote $a^{k}=V_{\alpha_{k} \beta_{k}}\left(x^{k}\right) /\left(\beta_{k}-\alpha_{k}\right)$. By (38), we have $a^{k} \leq \eta_{k}+\lambda_{k} a^{k-1}$ for $k=1,2, \ldots$ and it follows from (37) that $a^{k} \rightarrow 0$ ([16], Lemma 3). For each $k \in\{1,2, \ldots\}$, we have from Lemma 8 that (32) and (33) hold with $\alpha=$ $\alpha_{k}, \beta=\beta_{k}, x=x^{k}$. This together with $e_{\alpha_{k}, \beta_{k}}\left(x^{k}\right) \leq \varepsilon^{k}$ and $\left\|y_{\alpha_{k}}\left(x^{k}\right)-y_{\beta_{k}}\left(x^{k}\right)\right\| \leq \operatorname{diam}(X)$ yields

$$
\begin{aligned}
& \left\|x^{k}-y_{\beta_{k}}\left(x^{k}\right)\right\| \leq \sqrt{2 a^{k}}, \\
& \gamma^{k} \leq V_{\alpha_{k}}\left(x^{k}\right) \geq \gamma^{k}+\varepsilon^{k}+\frac{\alpha_{k} \operatorname{diam}(X)^{2}}{2},
\end{aligned}
$$

where $\gamma^{k}=\sum_{v=1}^{N}\left[\theta_{v}\left(x^{k, v}, x^{k,-v}\right)-\theta_{v}\left(y_{\beta_{k}}^{v}\left(x^{k}\right), x^{k,-v}\right)\right]-$ $(\alpha / 2)\left\|x^{k}-y_{\beta_{k}}\left(x^{k}\right)\right\|^{2}$ and $\operatorname{diam}(X)=\max _{x, y \in X}\|x-y\|$. Since $a^{k} \rightarrow 0$, the first inequality in (39) implies $\left\{x^{k}\right\}$ is bounded. Moreover, this also implies $r^{k} \rightarrow 0$.
Since $\alpha_{k} \rightarrow 0$, the last two inequalities in (39) yield $V_{\alpha_{k}}\left(x^{k}\right) \rightarrow 0$. Since for each $y \in X$, we have $V_{\alpha_{k}}\left(x^{k}\right) \geq$ $\Psi(x, y)-\left(\alpha_{k} / 2\right)\left\|x^{k}-y\right\|^{2}$, and this yields $0 \geq \Psi\left(x^{\infty}, y\right)$ for each cluster point $x^{\infty}$ of $\left\{x^{k}\right\}$. Thus, each cluster point $x^{\infty}$ is a normalized Nash equilibrium of the GNEP. This completes the proof.

Algorithm 11. Choose any $x^{0} \in R^{n}$, any $\beta_{0}>\alpha_{0}$, and two sequences of nonnegative numbers $\rho_{k}, \eta_{k}, k=1,2 \ldots$ such that

$$
\eta_{k}+\rho_{k}>0 \quad \forall k, \quad \sum_{k=1}^{\infty} \rho_{k}<\infty, \quad \sum_{k=1}^{\infty} \eta_{k}<\infty .
$$

Choose any continuous function $\phi: R_{+} \rightarrow R_{+}$with $\phi(t)=$ $0 \Leftrightarrow t=0$. For $k=1,2, \ldots$, we iterate the following.

Iteration $k$. Choose any $0<\alpha_{k} \leq(1 / 2) \alpha_{k-1}$ and then choose $\beta_{k} \geq 2 \beta_{k-1}$ satisfying

$$
\frac{V_{\alpha_{k} \beta_{k}}\left(x^{k-1}\right)}{\beta_{k}-\alpha_{k}} \leq\left(1+\rho_{k}\right) \frac{V_{\alpha_{k-1} \beta_{k-1}}\left(x^{k-1}\right)}{\beta_{k-1}-\alpha_{k-1}}+\eta_{k} .
$$

Apply a descent method to the unconstrained minimization of the function $V_{\alpha_{k} \beta_{k}}\left(x^{k}\right)$ with $x^{k-1}$ as the starting point. We assume the descent method has the property that the amount of descent achieved at $x$ per step is bounded away from zero whenever $x$ is bounded and $\left\|\nabla V_{\alpha_{k} \beta_{k}}(x)\right\|$ is bounded away from zero. Then, either the method in a finite number of steps generates an $x$ satisfying

$$
\left\|\nabla V_{\alpha_{k} \beta_{k}}(x)\right\| \leq \phi\left(\frac{V_{\alpha_{k} \beta_{k}}(x)}{\beta_{k}-\alpha_{k}}\right),
$$

which we denote by $x^{k}$, or else $V_{\alpha_{k} \beta_{k}}(x)$ must decrease towards zero, in which case any cluster point of $x$ solves the GNEP.

Theorem 12. Assume $X$ is bounded. Let $\left\{x^{k}, \alpha_{k}, \beta_{k}\right.$, $\left.\rho_{k}, \eta_{k}\right\}_{k=0,1,2, \ldots}$ be generated by Algorithm 11 .

(a) Suppose $x^{k}$ is obtained for all $k$. Then, $\left\{x^{k}\right\}$ is bounded; $\beta_{k} \rightarrow \infty ; \alpha_{k} \rightarrow 0$, and every cluster point of $\left\{x^{k}\right\}$ is a normalized Nash equilibrium of the GNEP.

(b) Suppose $x^{k}$ is not obtained for somek. Then, the descent method generates a bounded sequence of $x$ with $V_{\alpha_{k} \beta_{k}}(x) \rightarrow 0$ so every cluster point of $x$ solves the GNEP.

Proof. (a) Since we use a descent method at iteration $k$ to obtain $x^{k}$ from $x^{k-1}$, then $V_{\alpha_{k} \beta_{k}}\left(x^{k}\right) \leq V_{\alpha_{k} \beta_{k}}\left(x^{k-1}\right)$, so (41) yields

$$
\frac{V_{\alpha_{k} \beta_{k}}\left(x^{k}\right)}{\beta_{k}-\alpha_{k}} \leq\left(1+\rho_{k}\right) \frac{V_{\alpha_{k-1} \beta_{k-1}}\left(x^{k-1}\right)}{\beta_{k-1}-\alpha_{k-1}}+\eta_{k} \text {. }
$$

Denote $a^{k}=V_{\alpha_{k} \beta_{k}}\left(x^{k}\right) /\left(\beta_{k}-\alpha_{k}\right)$. This can then be written as $a^{k} \leq\left(1+\rho_{k}\right) a^{k-1}+\eta_{k}$ for $k=1,2, \ldots$ Using $a^{k} \geq 0$ and (41), it follows that the sequence $\left\{a^{k}\right\}$ converges to some $\bar{a} \geq 0$ ([16], Lemma 2). Since (32) implies

$$
\left\|x^{k}-y_{\beta_{k}}\left(x^{k}\right)\right\| \leq \sqrt{2 a^{k}}, \quad \forall k
$$

the sequence $\left\{x^{k}\right\}$ is bounded. 
We claim that $\bar{a}=0$. Suppose the contrary. Then for all $k$ sufficiently large, it holds that $a^{k} \geq \bar{a} / 2$. Then,

$$
\begin{aligned}
\frac{\bar{a}}{2} \leq & \frac{\sum_{v=1}^{N}\left[\theta_{v}\left(y_{\beta_{k}}^{v}\left(x^{k}\right), x^{k,-v}\right)-\theta_{v}\left(y_{\alpha_{k}}^{v}\left(x^{k}\right), x^{k,-v}\right)\right]}{\beta_{k}-\alpha_{k}} \\
& -\frac{\left(\alpha_{k} / 2\right)\left\|x^{k}-y_{\alpha_{k}}\left(x^{k}\right)\right\|^{2}+\left(\beta_{k} / 2\right)\left\|x^{k}-y_{\beta_{k}}\left(x^{k}\right)\right\|^{2}}{\beta_{k}-\alpha_{k}}
\end{aligned}
$$

Since, by the construction of the algorithm, $\beta_{k} \rightarrow \infty$ and $\alpha_{k} \rightarrow 0$, and $\left\{x^{k}\right\}$ is bounded (as are $y_{\alpha_{k}}\left(x^{k}\right)$ and $y_{\beta_{k}}\left(x^{k}\right)$ ), we get

$$
\begin{gathered}
0<\frac{\bar{a}}{2} \leq \liminf _{k \rightarrow \infty}\left\|x^{k}-y_{\beta_{k}}\left(x^{k}\right)\right\|^{2} . \\
\text { Then } \lim _{k \rightarrow \infty}\left\|x^{k}-y_{\beta_{k}}\left(x^{k}\right)\right\| \beta_{k}=\infty \text {, so } \\
\lim _{k \rightarrow \infty}\left\|\nabla V_{\alpha_{k}, \beta_{k}}\left(x^{k}\right)\right\|=\infty .
\end{gathered}
$$

Since $x^{k}$ satisfies (42), $\left\|\nabla V_{\alpha_{k}, \beta_{k}}\left(x^{k}\right)\right\| \leq \phi\left(a^{k}\right)$ for all $k$, this contradicts convergence of $\left\{\phi\left(a^{k}\right)\right\}$ (recall that $\phi$ is a continuous function). Hence, $\bar{a}=0$. For each $k \in\{1,2, \ldots\}$, we have from Lemma 8 that (33) holds with $\alpha=\alpha_{k}, \beta=\beta_{k}$, $x=x^{k}$ and from the fact $e_{\alpha \beta}(x) \geq 0$ that

$$
e_{\alpha_{k} \beta_{k}}\left(x^{k}\right) \leq \varepsilon^{k}:=\nabla V_{\alpha_{k} \beta_{k}}\left(x^{k}\right)^{T}\left(y_{\beta_{k}}\left(x^{k}\right)-y_{\alpha_{k}}\left(x^{k}\right)\right) \text {. }
$$

This, together with $\left\|y_{\alpha_{k}}\left(x^{k}\right)-y_{\beta_{k}}\left(x^{k}\right)\right\| \leq \operatorname{diam}(X)$, yields

$$
\gamma^{k} \leq V_{\alpha}(x) \leq \gamma^{k}+\varepsilon^{k}+\frac{\alpha_{k} \operatorname{diam}(X)^{2}}{2},
$$

where $\gamma^{k}=\sum_{v=1}^{N}\left[\theta_{v}\left(x^{k, v}, x^{k,-v}\right)-\theta_{v}\left(y_{\beta_{k}}\left(x^{k}\right)^{v}, x^{k,-v}\right)\right]-$ $\left(\alpha_{k} / 2\right)\left\|x^{k}-y_{\beta_{k}}\left(x^{k}\right)\right\|^{2}$ and $\operatorname{diam}(X)=\max _{x, y \in X}\|x-y\|$. Since $a^{k} \rightarrow 0$, (44) implies $\left\{x^{k}\right\}$ is bounded. Moreover, (44) implies $\gamma^{k} \rightarrow 0$. Also, we have $\left\|\nabla V_{\alpha_{k} \beta_{k}}\left(x^{k}\right)\right\| \leq \phi\left(a^{k}\right) \rightarrow 0$, so $\varepsilon^{k} \rightarrow 0$.

From the facts that $\alpha_{k} \rightarrow 0$, (49), $\gamma^{k} \rightarrow 0$ and $\varepsilon^{k} \rightarrow 0$, we get $V_{\alpha_{k}}\left(x^{k}\right) \rightarrow 0$. Since for each $y \in X$, we have from the definition of $V_{\alpha}(x)$ that

$$
V_{\alpha_{k}}\left(x^{k}\right) \geq \Psi(x, y)-\frac{\alpha_{k}}{2}\left\|x^{k}-y\right\|^{2}
$$

which yields $0 \geq \Psi\left(x^{\infty}, y\right)$ for each cluster point $x^{\infty}$ of $\left\{x^{k}\right\}$. Thus, each cluster point $x^{\infty}$ is a normalized Nash equilibrium of the GNEP.

(b) It is easy to proof that $V_{\alpha_{k} \beta_{k}}(x) \rightarrow 0$. Hence $x^{\infty}$ is a normalized Nash equilibrium of the GNEP.

The proof is completed.

\section{Acknowledgments}

This research was partly supported by the National Natural Science Foundation of China $(11271226,10971118)$ and the Promotive Research Fund for Excellent Young and MiddleAged Scientists of Shandong Province (BS2010SF010).

\section{References}

[1] A. Dreves and C. Kanzow, "Nonsmooth optimization reformulations characterizing all solutions of jointly convex generalized Nash equilibrium problems," Computational Optimization and Applications, vol. 50, no. 1, pp. 23-48, 2011.

[2] A. Dreves, C. Kanzow, and O. Stein, "Nonsmooth optimization reformulations of player convex generalized Nash equilibrium problems," Journal of Global Optimization, vol. 53, no. 4, pp. 587-614, 2012.

[3] F. Facchinei and C. Kanzow, "Generalized Nash equilibrium problems," Annals of Operations Research, vol. 175, pp. 177-211, 2010.

[4] F. Facchinei, A. Fischer, and V. Piccialli, "On generalized Nash games and variational inequalities," Operations Research Letters, vol. 35, no. 2, pp. 159-164, 2007.

[5] F. Facchinei and C. Kanzow, "Generalized Nash equilibrium problems," A Quarterly Journal of Operations Research, vol. 5, no. 3, pp. 173-210, 2007.

[6] D. Han, H. Zhang, G. Qian, and L. Xu, "An improved two-step method for solving generalized Nash equilibrium problems," European Journal of Operational Research, vol. 216, no. 3, pp. 613-623, 2012.

[7] P. T. Harker, "Generalized Nash games and quasi-variational inequalities," European Journal of Operational Research, vol. 54, no. 1, pp. 81-94, 1991.

[8] A. von Heusinger and C. Kanzow, "Optimization reformulations of the generalized Nash equilibrium problem using Nikaido-Isoda-type functions," Computational Optimization and Applications, vol. 43, no. 3, pp. 353-377, 2009.

[9] B. Panicucci, M. Pappalardo, and M. Passacantando, "On solving generalized Nash equilibrium problems via optimization," Optimization Letters, vol. 3, no. 3, pp. 419-435, 2009.

[10] B. Qu and J. G. Jiang, "On the computation of normalized nash equilibrium for generalized nash equilibrium problem," Journal of Convergence Information Technology, vol. 7, no. 22, pp. 16-21, 2012.

[11] J. Zhang, B. Qu, and N. Xiu, "Some projection-like methods for the generalized Nash equilibria," Computational Optimization and Applications, vol. 45, no. 1, pp. 89-109, 2010.

[12] S. D. Flåm and A. S. Antipin, "Equilibrium programming using proximal-like algorithms," Mathematical Programming, vol. 78, no. 1, pp. 29-41, 1997.

[13] S. D. Flåm and A. Ruszczyński, "Noncooperative convex games: computing equilibrium by partial regularization," Working Paper 94-42, Laxenburg, Austria, 1994.

[14] M. V. Solodov and P. Tseng, "Some methods based on the Dgap function for solving monotone variational inequalities," Computational Optimization and Applications, vol. 17, no. 2-3, pp. 255-277, 2000.

[15] B. Qu, C. Y. Wang, and J. Z. Zhang, "Convergence and error bound of a method for solving variational inequality problems via the generalized D-gap function," Journal of Optimization Theory and Applications, vol. 119, no. 3, pp. 535-552, 2003.

[16] B. T. Polyak, Introduction to Optimization, Translations Series in Mathematics and Engineering, Optimization Software, New York, NY, USA, 1987. 


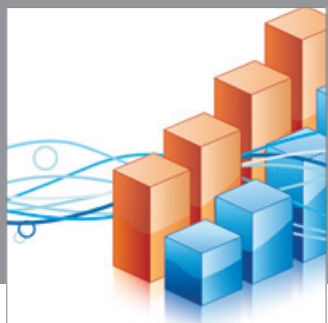

Advances in

Operations Research

mansans

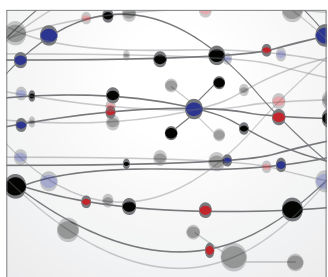

The Scientific World Journal
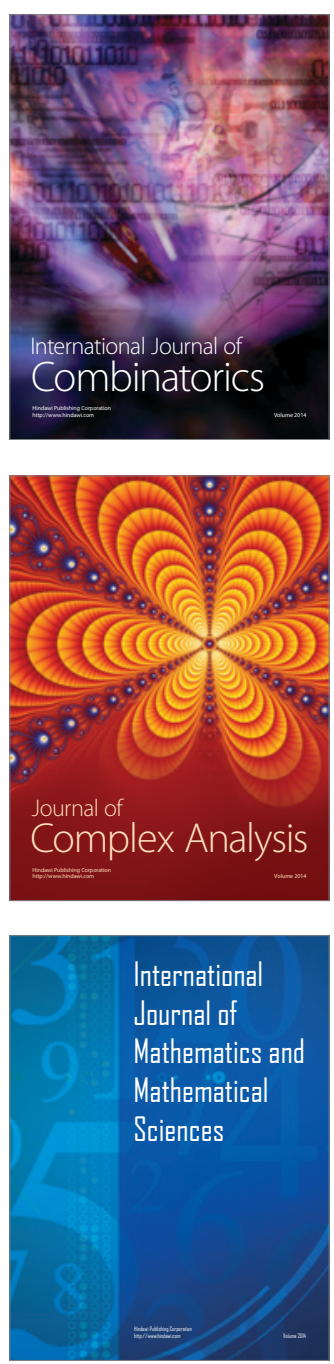
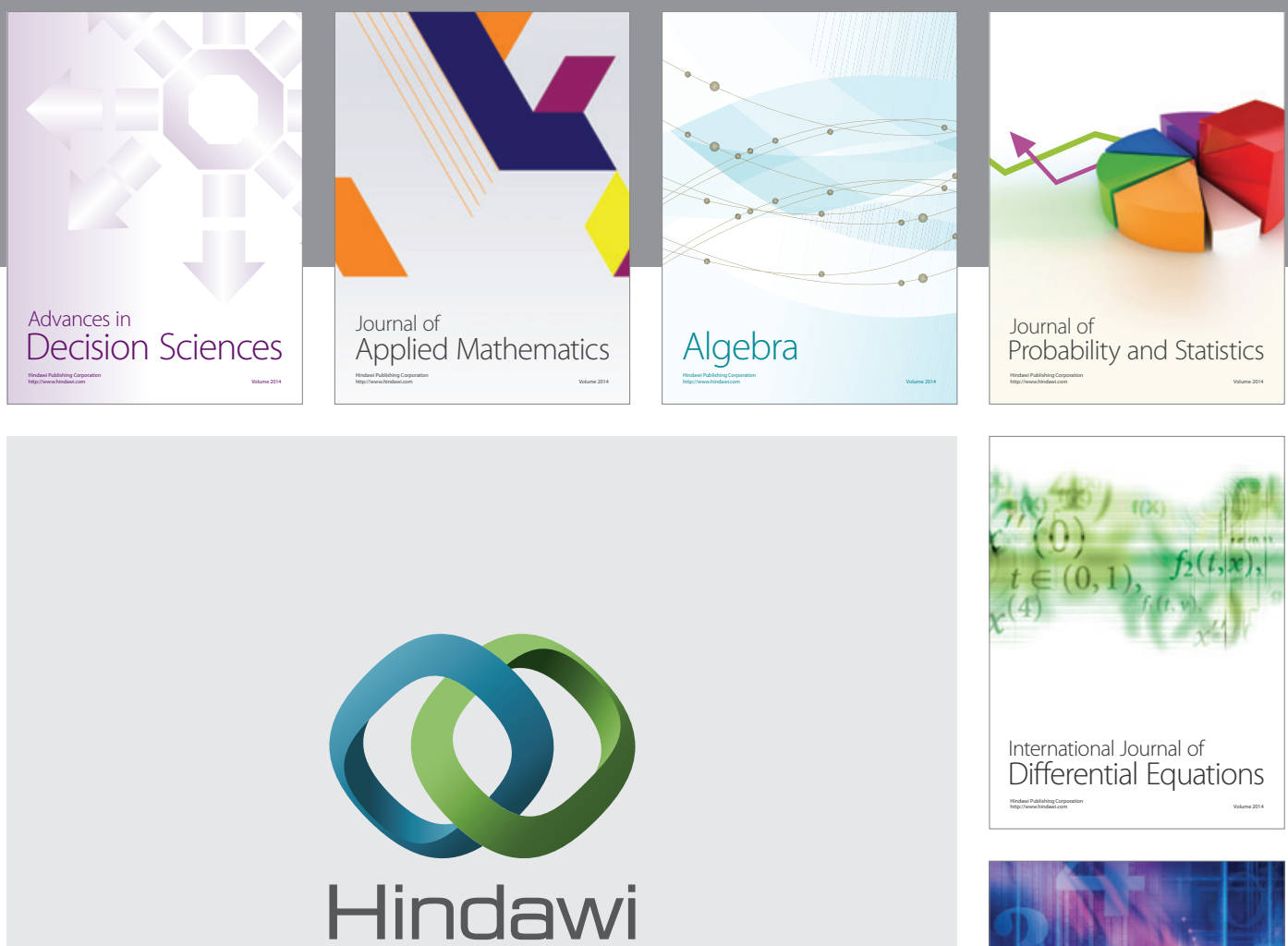

Submit your manuscripts at http://www.hindawi.com
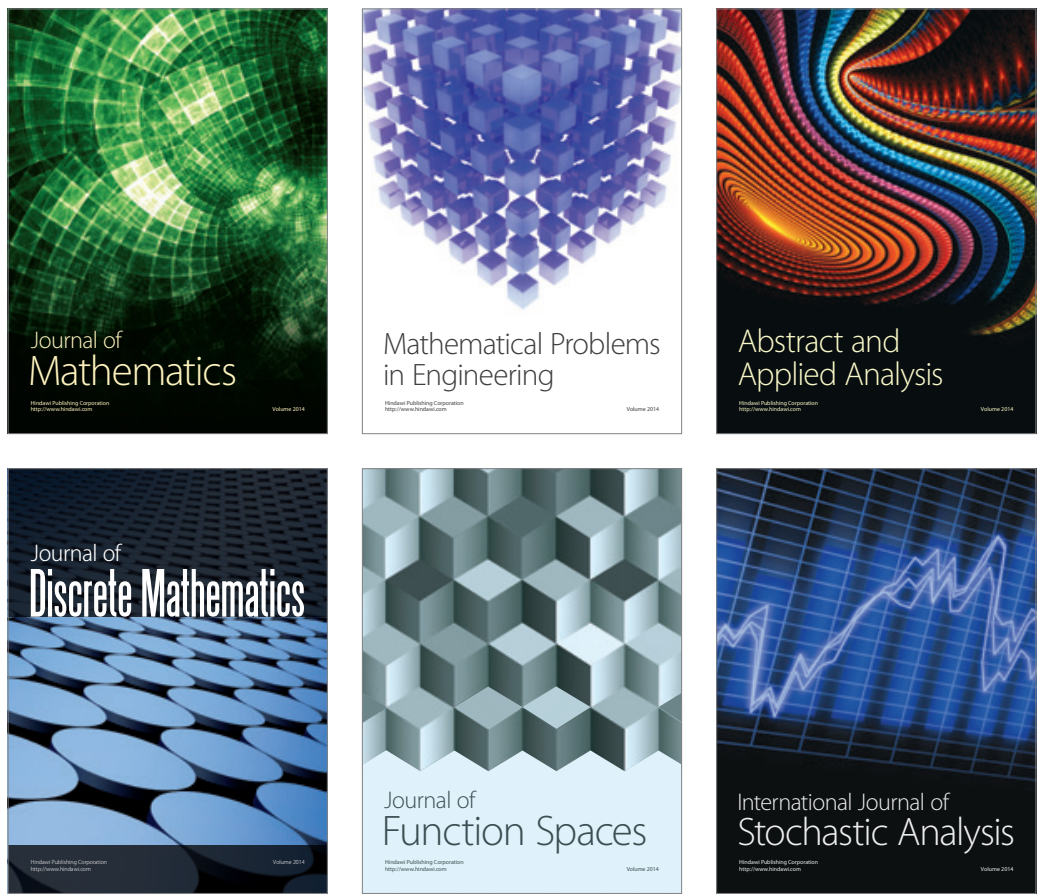

Journal of

Function Spaces

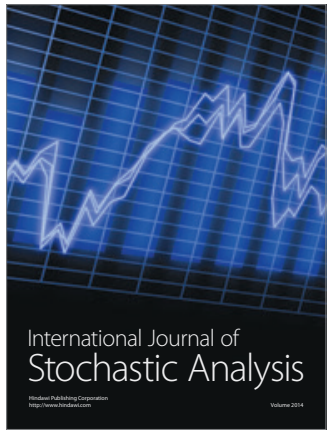

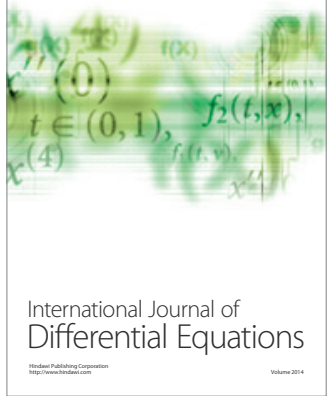
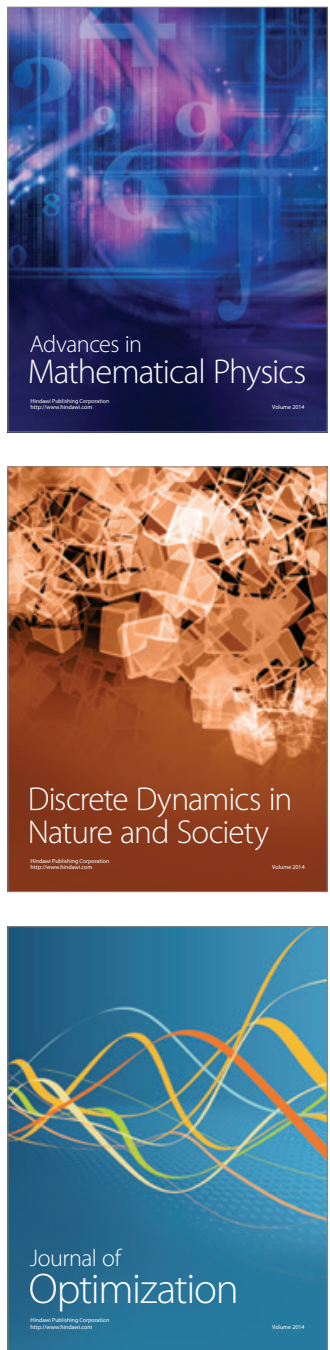